\title{
Validación de una plataforma de evaluación formativa de la competencia digital docente en tiempos de Covid-19
}

\section{(Validation of a Platform for Formative Assessment of Teacher Digital Competence in Times of COVID-19)}

\author{
Virginia Viñoles-Cosentino \\ Francesc Marc Esteve-Mon \\ $\mathrm{M}^{\mathrm{a}}$ Ángeles Llopis-Nebot \\ Jordi Adell-Segura \\ Universitat Jaume I, UJI (España)
}

DOI: https://doi.org/10.5944/ried.24.2.29102

\section{Cómo referenciar este artículo:}

Viñoles-Cosentino, V., Esteve-Mon, F. M., Llopis-Nebot, M. A., y Adell-Segura, J. (2021). Validación de una plataforma de evaluación formativa de la competencia digital docente en tiempos de Covid-19. RIED. Revista Iberoamericana de Educación a Distancia, 24(2), pp. 87-106. https://doi.org/10.5944/ ried.24.2.29102

\section{Resumen}

La progresiva incorporación de las tecnologías digitales en la educación superior ha puesto de manifiesto la necesaria competencia digital docente (CDD). Una competencia que no solo implica conocimientos técnicos, sino también pedagógicos y de empoderamiento del estudiante. En la última década, algunas universidades han revisado sus planes de digitalización o transformación digital, alineando sus estrategias y políticas formativas en el desarrollo de esta competencia, algo que todavía se ha vuelto más necesario, si cabe, durante la crisis sanitaria derivada de la Covid-19. El presente artículo, que se enmarca en un proyecto de investigación basado en el diseño de una plataforma de autoevaluación y formación en CDD, profundiza de manera cualitativa en la valoración del profesorado acerca de esta competencia, en la utilidad de esta plataforma de evaluación y formación, y en la percepción sobre el efecto de la pandemia en este ámbito. Las entrevistas semiestructuradas en profundidad de docentes universitarios, gestores universitarios y técnicos en tecnología educativa $(n=15)$, fuente principal de información, permiten profundizar en el análisis de la CDD y generar una serie de principios de diseño útiles para el desarrollo de este tipo de plataformas y para el diseño de estrategias y políticas de formación digital del profesorado. 
Palabras clave: competencia digital docente; enseñanza superior; formación docente; evaluación educativa.

\begin{abstract}
The progressive incorporation of digital technologies in higher education has highlighted the need for teacher digital competence (TDC). A competence that not only implies technical knowledge, but also pedagogical knowledge and student empowerment. Over the last decade, some universities have revised their digitalisation or digital transformation plans, aligning their training strategies and policies with the development of this competence, something which has become even more necessary, if possible, during the health crisis resulting from Covid-19. This article, which is part of a research project based on the design of a selfassessment and training platform in TDC, teacher digital competence, delves qualitatively into teachers' assessment of this competence, the usefulness of this assessment and training platform, and the perception of the effect of the pandemic in this area. Semi-structured-indepth-interviews with university teachers, university managers and educational technology technicians $(n=15)$, the main source of information, allow for an in-depth analysis of the TDC and generate a series of design principles useful for the development of this type of platforms and for the design of strategies and policies for the digital teacher training.
\end{abstract}

Keywords: teacher digital competence; higher education; teacher training; educational assessment.

En la última década se ha evidenciado la importancia de impulsar mecanismos de transformación digital en la universidad, que incidan en los procesos de enseñanzaaprendizaje (Marshall, 2018). Este hecho, ha quedado más patente durante el 2020 debido a la crisis derivada por la Covid-19, la cual ha generado múltiples situaciones formativas disruptivas, y una proliferación sin precedentes de actividades docentes en espacios semipresenciales, híbridos o totalmente en línea (Marinoni et al., 2020).

La inclusión de tecnologías digitales (TD) en el ámbito educativo exige la adaptación y desarrollo de nuevas competencias docentes (López et al., 2016). Para aprovechar el potencial de las herramientas digitales en los procesos formativos, así como para gestionar estas nuevas situaciones de aprendizaje enriquecidas por las TD), es necesario que el profesorado universitario disponga de una adecuada competencia digital docente (CDD) (Fernández-Batanero et al., 2020). Esta competencia comprende el conjunto de habilidades, conocimientos y actitudes que deben poner en juego los docentes para facilitar el aprendizaje de los alumnos a través del diseño y aplicación de prácticas formativas ajustadas al contexto digital (Gisbert et al., 2016). La CDD permite al profesorado responder a los desafíos de la sociedad digital, potenciar el proceso de enseñanza-aprendizaje y continuar desarrollándose profesionalmente, colaborando con otros colegas y actualizándose; e incrementar 
también la competencia digital (CD) de su estudiantado, una competencia clave para su empoderamiento y ciudadanía digital (Esteve et al., 2020a).

Sin embargo, como plantea Pettersson (2018), el desarrollo de la CDD no puede ser responsabilidad exclusiva de los docentes. Las universidades deben responder a estas nuevas demandas con una adecuada formación digital docente, algo que no siempre se contempla adecuadamente en los planes de formación del profesorado (Marín et al., 2012).

El trabajo que se presenta a continuación se enmarca en un proyecto de investigación que se centra en analizar la CDD del profesorado universitario y diseñar una plataforma formativa que facilite su desarrollo. Los objetivos planteados son, profundizar en la percepción del nivel de CDD, conocer la valoración de la herramienta formativa a partir de las distintas visiones de sus potenciales usuarios, así como analizar la posible incidencia de la pandemia de la Covid-19 en esta competencia y en la formación digital del profesorado.

\section{LA COMPETENCIA DIGITAL DOCENTE DEL PROFESORADO UNIVERSITARIO}

La CDD es un constructo relativamente reciente, y ello dificulta hallar una definición teórica unificada, en cambio, existen numerosos marcos conceptuales y modelos, a nivel internacional, que han tratado de caracterizarla (Caena y Redecker, 2019). Según Krumsvik (2012) puede definirse como la capacidad del docente para usar las tecnologías con un adecuado criterio pedagógico y didáctico, frente a unos contenidos concretos, así ser consciente de estas e incluirlas como parte de sus estrategias de aprendizaje permanente. Según autores como Hall et al. (2014), la CDD comprende el conjunto de habilidades, conocimientos y actitudes necesarias para favorecer el aprendizaje de sus estudiantes, en una sociedad cada vez más digitalizada, transformando las prácticas de aula, y enriqueciendo su propio desarrollo e identidad como docente.

A pesar de la existencia de múltiples marcos de CDD, la mayor parte de estos se centran en etapas docentes preuniversitarias (Durán et al., 2016). Uno de los más conocidos es el Marco Europeo para docentes digitalmente competentes (DigCompEdu), elaborado por el Joint Research Center de la Comisión Europea (Redecker y Punie, 2017), y que sintetiza esta competencia en 6 áreas: (1) compromiso profesional, es decir, la comunicación y colaboración profesional, o la práctica reflexiva y desarrollo profesional; (2) la selección, creación y gestión de recursos digitales; (3) la enseñanza, tutorización y aprendizaje, (4) el diseño de estrategias de evaluación y retroalimentación al estudiante; (5) el empoderamiento, personalización e inclusión del estudiante, y (6) el desarrollo de la propia CD de sus estudiantes. Si bien se trata de un marco amplio, usado en niveles no universitarios, ha sido adaptado y utilizado en algunas universidades (Alarcón et al., 2020; Tourón et al., 2018). 
En los últimos años han aparecido distintos estudios que analizan la CDD del profesorado universitario (Esteve et al., 2020a). Gran parte de estas investigaciones evidencian que el profesorado posee, en general, un nivel medio-alto en habilidades técnicas, destacando aspectos informacionales y comunicativos (Rangel y Peñalosa, 2013). Sin embargo, se detectan niveles intermedios y bajos en la dimensión pedagógica de esta competencia, tanto en la planificación y el diseño de situaciones de aprendizaje con TD como en la evaluación del estudiante (Pozos y Tejada, 2018) e igualmente se evidencian niveles medio-bajos en el uso de las TD para el desarrollo profesional, como puede ser la participación en redes y foros, o el uso de repositorios educativos (Orozco et al., 2016). Estas deficiencias pueden deberse a la falta de estrategias universitarias para la integración de las tecnologías en las aulas, lo cual impacta negativamente en la percepción de los propios docentes, especialmente entre los más innovadores (Mercader y Gairín, 2020).

\section{ESTRATEGIAS Y POLÍTICAS UNIVERSITARIAS PARA EL DESARROLLO DE LA COMPETENCIA DIGITAL DOCENTE}

Ante la necesidad de atender a las necesidades de los docentes en educación superior, las universidades, durante la última década, han ido adquiriendo el compromiso de adaptar su propuesta formativa a esta realidad. Sin embargo, los itinerarios formativos dirigidos al profesorado, resultan muchas veces insuficientes, descontextualizados y en algunos casos, con poca posibilidad de aplicarse en el aula (Halász, 2019). Las principales limitaciones en el establecimiento de planes de formación docente han sido (1) presentar prácticas no adecuadas o inconsistentes respecto a los objetivos perseguidos; (2) carecer de visión crítica respecto a la valoración de resultados; (3) partir de enfoques limitados, que no contemplan la complejidad del cambio educativo ni se adaptan a la realidad del contexto y (4) la falta de apoyo institucional (Ion y Cano, 2012; Ruè, 2015).

$\mathrm{Al}$ revisar las distintas iniciativas de formación y digitalización docente en educación superior, se encuentran tres tipos de estrategias principales. Por un lado, algunas universidades como la de Bergen (2016), Athabasca (2018) o Auckland (2018) centran sus esfuerzos en implementar mejoras en las infraestructuras tecnológicas del campus, promover la cultura del cambio y favorecer el uso de servicios digitales. Otras universidades centran sus políticas formativas en el desarrollo de la CD de su alumnado, tal sería el caso de la Universidad Católica de Lovaina (2015), que promueve la creación de recursos educativos libres, de MOOC, y de servicios de apoyo para la formación y el aprendizaje digital. El tercer enfoque, llevado a cabo en universidades como la de Aalborg (2018) o Ginebra (2019), implementaron en su plan estratégico de digitalización una serie de acciones para el desarrollo de la CD de toda la comunidad universitaria, apoyando la creación de recursos digitales para la enseñanza y el aprendizaje tales como herramientas de autoevaluación de la CD y de formación presencial y en línea, en función de las carencias detectadas. 


\section{CONTEXTO DE LA INVESTIGACIÓN}

La presente investigación se ha llevado a cabo en el contexto de la Universitat Jaume I (UJI) de Castellón (España), que en 2019 inició un proceso de diagnóstico y definición de su estrategia de digitalización, denominado Plan UJI Digital (Universitat Jaume I, 2020). Este plan recoge una serie de acciones estratégicas enmarcadas en 4 ejes: (1) la CD de la comunidad universitaria; (2) la educación digital y en línea; (3) la investigación y la transferencia digital; y (4) los procesos y servicios que facilitan la digitalización universitaria. Entre las acciones relacionadas con la mejora de la CDD destacan, concienciar al profesorado sobre la importancia de dicha competencia en su desarrollo profesional, proporcionar elementos de evaluación diagnóstica y continuada, e implementar itinerarios y recursos formativos para su desarrollo. Tomando como referencia el marco europeo DigCompEdu (Redecker y Punie, 2017), se ha diseñado el plan de formación del profesorado para el curso 2020-2021, y a su vez han surgido diferentes proyectos de investigación buscando desarrollar, en primer lugar, una plataforma de autoevaluación digital docente y, en segundo lugar, una web bajo la cual se centralizan y organizan los recursos formativos.

La plataforma de autoevaluación diagnóstica (http://cdd.uji.es) cuenta con un cuestionario de autopercepción a través del cual el profesorado conoce su nivel de CDD, adaptando el marco DigCompEdu al contexto particular de la institución. Esta herramienta fue diseñada en el 2019, y validada durante 2020 con una muestra representativa de 558 docentes universitarios, de los diferentes ámbitos de conocimiento y categorías profesionales (Llopis et al., 2020). Para garantizar que los ítems eran comprendidos, previamente se validaron mediante grupos focales y entrevistas cognitivas (Drennan, 2003) y se administró a un grupo piloto, obteniéndose un índice de fiabilidad alto $(\alpha=0.94)$ según el alfa de Cronbach. Los resultados obtenidos sugieren que el profesorado posee un nivel medio-alto en su competencia profesional digital, relacionada con habilidades comunicativas y de organización personal. Con unas puntuaciones ligeramente inferiores, se encuentran áreas relacionadas con el empoderamiento digital del alumnado o el ámbito pedagógico, en relación a la creación de recursos digitales o el uso de las TD para la enseñanza y la evaluación del aprendizaje, debiéndose incidir en su formación.

En la segunda parte del proyecto se desarrolló la plataforma de recursos de formación en CDD del profesorado universitario (http://cdd.uji.es/recursos). Esta herramienta recoge propuestas formativas de la universidad (cursos, seminarios y talleres) y permite establecer un filtrado o bien, en función de las áreas de la CDD del modelo DigCompEdu, o bien a partir de características que determine el propio usuario, como puede ser el nivel de complejidad (básico, intermedio y avanzado) o la modalidad de la formación (presencial, virtual y semipresencial) (Esteve et al., 2020b). 


\section{METODOLOGÍA}

La investigación se llevó a cabo en la UJI y se enmarca dentro de los estudios de investigación para el diseño educativo (Educational-Design Research, EDR). Siguiendo los planteamientos del EDR (Plomp y Nieveen, 2009), en esta fase del proyecto se pretende explorar en profundidad los criterios de calidad (validez, practicidad y efectividad) aplicados al desarrollo de la plataforma de evaluación de la CDD y de los recursos educativos para la formación digital del profesorado. Para ello, y partiendo de los objetivos planteados, este estudio trata de explorar tres preguntas principales:

1. ¿Hasta qué punto es válido el nivel de CDD del profesorado universitario obtenido en la plataforma de autoevaluación diagnóstica?

2. ¿Hasta qué punto resulta de utilidad la plataforma de recursos de formación en CDD de la universidad?

3. ¿Cómo ha afectado la pandemia derivada de la Covid-19 en la percepción del profesorado universitario sobre su CDD?

A través de entrevistas semiestructuradas (Corbin y Strauss, 2015), se exploran las cuestiones anteriores en una muestra formada por 15 miembros de la comunidad universitaria: (a) 9 docentes universitarios ("P1-9"), de diferentes áreas de conocimiento (1 de Ciencias de la Salud y Comportamiento, 2 de Ingeniería y Arquitectura, 2 de Ciencias Sociales, 2 de Artes y Humanidades, y 2 de Ciencias Jurídicas y Económicas) y distintas categorías profesionales (1 Catedrática, 2 Profesores Titulares, 1 Contratado Doctor, 3 Ayudantes Doctores y 2 Asociados); (b) 3 gestores ("G1-3"), responsables de políticas de la universidad, del ámbito de la formación, la investigación y la internacionalización; y (c) 3 técnicos ("T1-3"), responsables de los planes de formación digital del profesorado. El número de participantes y su tipología fue elegida de manera deliberada (purposive sampling), siguiendo criterios de muestreo por intensidad (intensity sampling) e informantes clave (Tessmer, 1993), por el cual, sin ser una muestra estadísticamente representativa de sujetos, se recogen situaciones extremas que manifiesten con gran intensidad el fenómeno en estudio, permitiendo su representatividad o comparabilidad (Teddlie y $\mathrm{Yu}, 2007$ ), adecuada en estudios cualitativos y mixtos, así como en procesos de construcción de teoría fundamentada. En la selección de la muestra se establecieron tres criterios de inclusión: (1) que hubieran participado, de manera voluntaria, en la fase de autoevaluación de su CDD, previa a la Covid-19; (2) que utilizaran las TD en su docencia universitaria; y (3) que impartieran docencia posteriormente al confinamiento por la Covid-19.

Siguiendo a Tessmer (1993), las entrevistas incluyeron tres secciones: (1) una inicial, centrada en contrastar los resultados de la autoevaluación de la CDD del profesorado universitario de la UJI, a partir de la revisión de estos; (2) una segunda, 
con cinco preguntas abiertas centradas en la usabilidad, claridad y practicidad del portal de recursos para la formación digital; y (3) una última sección con preguntas abiertas acerca de las políticas universitarias de formación digital, y el efecto de la Covid-19 en la CDD. Las entrevistas duraron un promedio de 40 minutos y se realizaron durante el mes de noviembre de 2020. Todas fueron realizadas por videoconferencia, grabadas y codificadas, de manera simultánea, utilizando un enfoque deductivo basado en conceptos o codebook, a partir de las preguntas de investigación (Saldaña, 2015), y utilizando para ello el software de análisis cualitativo MAXQDA (versión 2018). El marco de conceptos fue diseñado con anterioridad a la realización de las entrevistas, discutido y ampliado posteriormente, para incluir aspectos surgidos en las mismas. Todos los desacuerdos en la codificación se resolvieron mediante discusión y, a partir de esta, se seleccionaron las respuestas coincidentes y su interpretación (Corbin y Strauss, 2015).

\section{RESULTADOS}

A continuación se detallan los principales resultados obtenidos, organizados según las preguntas de investigación. Los resultados se presentan mediante un análisis narrativo, acompañado de fragmentos y citas literales, contextualizadas e interpretadas (Rodríguez-Gómez et al., 1999), que permite ilustrar y entender mejor, desde la perspectiva de los propios participantes, el nivel de CDD, la utilidad de la plataforma formativa, y la posible incidencia de la pandemia.

\section{Nivel de competencia digital docente del profesorado universitario}

Uno de los aspectos más mencionados durante las entrevistas está relacionado con el ajuste entre la autopercepción del profesorado respecto a su CDD a partir de los resultados del cuestionario, en comparación con la realidad. Algunos participantes manifestaron que dicho nivel se ajustaba a lo que ellos percibían como nivel general de CDD en la universidad, sin embargo, otros participantes los consideraron bastante más elevados de lo que hubieran esperado.

"Sí que creo que la percepción de la mayoría de los docentes sea un nivel medio, porque ha habido recursos, la universidad sí que ha proporcionado recursos" (P1).

"Me da la sensación de que somos muy optimistas (...) El profesorado se ve muy bien, se ve de una manera muy complaciente" (P6).

"Creo que hay una parte importante de autoengaño en función de lo que yo conozco, a mí no me cuadra con todo lo que estoy viendo en el día a día, no lo identifico, sobre todo porque es muy alto"(P9). 
"Sí que me cuadra que un 20\% de la población del profesorado tenga un nivel bajo o muy bajo de competencia digital. Y un 80\% que lo tenga intermedio o alto también. Me sorprende que haya casi un $40 \%$ que digan que es alto, con lo cual tienen una muy buena percepción de sí mismos. Pero sí, yo creo que está bastante ajustado"(T2).

En cuanto a las áreas de competencias profesionales en el ámbito digital, se extrae que estas competencias se ven altamente favorecidas por las características de la universidad, se trata de una universidad joven, con adecuada infraestructura, y que ha implementado estrategias de formación digital desde tiempo atrás.

"En cuanto al compromiso profesional, competencias profesionales digitales, entiendo que sea tan alto porque la universidad ha hecho un gran esfuerzo en digitalización en estos últimos dos, tres años" (P4).

"Ahí sí que no me extraña porque en ese sentido nuestra universidad fue la primera en digitalizarse, al tener que utilizar las herramientas digitales el profesorado tiene que tener un mínimo, por eso salen un poco más alto las primeras y no tanto la de motivación a estudiantes" (G2).

Sin embargo, la mayor parte de los participantes señalan como demasiado elevados los resultados relativos a las competencias didácticas y empoderamiento del estudiantado, diferenciando entre lo que sería un cambio metodológico a través del uso de la tecnología de lo que es su mero uso instrumental para la docencia.

"Lo de competencias didácticas lo veo demasiado alto, pero hablo desde mi perspectiva personal. No nos ha dado tiempo a lograr eso creo yo, una cosa es que uses la tecnología y otra cosa que la uses con un método didáctico, que creo que es bastante distinto" ( $\mathrm{P2}$ ).

"No es que sea la gente que quiera engañar, sino que es la percepción que se tiene, que tú tengas un Aula Virtual y cuelgues lo mismo que antes dejabas en reprografía, eso no es" (P9).

"La parte de competencias de los estudiantes, empoderarlos sobre todo, las veo notablemente hinchadas, es la que veo más anormalmente alta" (T3).

Mayoritariamente, las personas entrevistadas atribuyen esta supuesta desviación de resultados a la voluntariedad de participación en la encuesta. Ello podría implicar que quienes participan creen tener nivel suficiente de CDD o bien están más comprometidas con su formación: 
"Hay que ver si la gente que no tiene competencia digital se atreve a hacer una encuesta de este tipo y mostrarlo. A lo mejor hay parte oculta de gente que le da vergüenza reconocerlo... aquí vienen los convencidos" (P2).

"He pensado que seguramente las 600 personas que contestamos precisamente eran, yo me pongo en esa línea, quienes tuvimos mucho compromiso (...) La gente que no estuvo implicada o no tiene mucho compromiso en este asunto pues seguramente tampoco hizo la encuesta" ( $P 7)$.

"Al ser sobre una base voluntaria, siempre tienes un sesgo, a lo mejor de ahí sale que tenga ese gran porcentaje de competencia alta. Así, esos datos sí que me cuadrarían" (G3).

No obstante, cuando se incide en si ven su propio nivel de CDD reflejado en los resultados, manifiestan de forma casi unánime que sí se identifican con ellos, tanto en el nivel general de competencia, como en la distribución por áreas de conocimiento.

"Sí que me siento reflejada, me sorprendió también en la autoevaluación el tener un poco más bajo el apartado de competencias de los estudiantes” (P3). "Por ejemplo a mí sí que me pasaba esto. Tenía como mucho mejores las dos primeras: competencias profesionales y competencias didácticas, comparado con el tema de empoderar al estudiantado, de facilitar las competencias digitales del estudiantado" $\left(P_{5}\right)$.

Finalmente, durante las entrevistas también se han sugerido nuevos aspectos a investigar, como la posibilidad de triangular los resultados incorporando la visión del estudiantado, o analizar la posible influencia del sesgo de género.

"El alumno no tiene esta percepción, no sé si habéis hecho la encuesta a los alumnos pero la distorsión sería tremenda" $(P 7)$.

"Hay otro tema, que me permito introducir, que es el de la brecha de género. Ahí hay como fuerzas contradictorias ino? Por un lado partimos de una brecha digital en las mujeres, general en la sociedad, que es verdad que en algunos aspectos se va superando. Pero en el ámbito educativo tengo mis dudas si no se da al contrario. La preocupación por la formación docente también tiene una brecha, pero al contrario, esto probablemente ha hecho que en general, aún superando la supuesta tecnofobia femenina, nos formásemos más en general" (P6).

"A lo mejor se puede encuestar al alumnado para ver qué tipo de percepción tienen. Es que creo que esto ha sido un antes y un después, ellos tienen una visión totalmente diferente seguramente" $\left(G_{3}\right)$. 


\section{Utilidad de la plataforma de recursos de formación en CDD}

La amplia mayoría de participantes encuentra útil contar con una plataforma como la desarrollada en el proyecto, que permita el autodiagnóstico de la CDD, y a partir de los resultados ofrezca recursos para la formación digital.

"Útil totalmente, porque ahí en función a tu nivel puedes elegir. Es útil porque te marca el nivel, la percepción que tengas de tu nivel te puede acercar a los cursos que más se ajustan" $(P 1)$.

"Me parece genial, porque no es lo mismo simplemente poner cursos de competencia digital, a hacerte una autoevaluación de tu competencia digital y ver donde fallas más" (P5).

"Me ha gustado que esté disponible la explicación de en qué consiste cada área. Me parecería útil que después de contestar la encuesta le sugiera qué cursos serían más útiles" (T1).

"Me parece muy práctico y claro. Lo ideal es intentar que el profesorado primero haga un autodiagnóstico y se conciencie, sepa en qué punto está y qué tiene para mejorar. Me parece súper práctico porque con áreas a mejorar y con filtros es muy fácil detectar la formación ofrecida para que pueda mejorar" (T2).

Además de la utilidad en sí, se destaca el valor formativo de la plataforma, como herramienta beneficiosa para la sensibilización acerca de la importancia de la CDD.

"Me parece interesante como repositorio de recursos, y además para formar en el metalenguaje adecuado cuando hablamos de competencia digital y qué implica la competencia digital" (P4).

Sobre los cursos de formación que se incluyen en la plataforma, diferentes participantes sugieren que sería interesante que los materiales pudieran estar disponibles y accesibles de manera permanente.

"Los cursos online en teoría no tienen fecha de caducidad. Entiendo que para la certificación o para lo que sea, sí, pero puedes acceder luego igualmente a los recursos. Los recursos tendrían que estar siempre, aunque los foros no vayan. La tutorización, sí, es importante en los cursos a distancia. Pero creo que los cursos deberían estar abiertos siempre" (P2).

"(Es importante) acercar toda esta formación al profesorado, la posibilidad de interactuar con todos esos recursos, que sea más cercana y más inmediata. Lo necesito aquí $y$ ahora, $y$ puedo acceder" (P4). 
"Iría más allá, que hubiera dentro de esta plataforma algún tipo de indicación a recursos estáticos, este curso se acabó pero puedes obtener más información aquí" (G1).

Entre los elementos de mejora del portal se cita, por un lado, la posibilidad de integrar un buscador abierto que permita localizar fácilmente el material, y por otro, la inclusión de un sistema de valoración o recomendación de las ofertas formativas.

"Esto tendrá éxito o no, si tú encuentras rápido lo que buscas. Eso de acumular información... yo lo que quiero es encontrar rápido la solución. Puede ayudar mucho poner lo más solicitado, como en Youtube, lo más pedido, que tenga más visitas. Si algo se ve muchas veces es que está bien hecho" (P2).

También se destaca la importancia de brindar más información sobre los recursos que se muestran a través del portal, así como una adecuada difusión para optimizar su alcance entre el profesorado.

"Me ha gustado que esté disponible la explicación de cada área, al profesor no le ayudan mucho las categorías si no se le explica y me parece muy útil que esté explicado para que vea que categorización hay" (T1).

"Me parece importante saber quién da un curso, por qué se especializan en eso. La parte ética también es importante para mí, quién está detrás"(P9).

"El tema es la publicidad, enterarse... en la universidad recibimos tantos emails, que se me escapan" (P5).

Un aspecto deseable para el portal tiene que ver con su carácter interactivo y bidireccional. Se menciona, por parte de los tres colectivos entrevistados, la posibilidad de que el profesorado haga llegar sus sugerencias y peticiones específicas de formación, así como la posibilidad de abrir espacios de intercambio de información y ayuda colaborativa entre usuarios.

"Le añadiría alguna opción para recabar peticiones del profesorado, entro y quiero formarme más sobre empoderamiento del alumnado y veo que está el nivel básico, y a lo mejor quisiera algo con nivel un poco más intermedioavanzado" (T2).

"Algún foro de gente que está haciendo los cursos. Al final aprendemos mucho porque preguntamos, compartimos. "Yo utilicé para tal cosa esto». Un foro estaría muy bien que genere interacción y comunicación con gente que está trabajando los mismos temas que nosotros, que podamos aportar y que nos aporten" (P1). 
"Es interesante un foro de comunicación para que ese tipo de experiencias y buenas prácticas se vean, es muy interesante saber qué hacen los demás. Te comparas, «yo esto no lo estoy haciendo me resultaría interesante»" (G1).

Finalmente, se reflexiona sobre algunos desafíos que plantea la formación docente universitaria, como es la heterogeneidad del profesorado que proviene de distintos ámbitos de conocimiento, y que puede tener necesidades formativas muy distintas, unido a la complejidad de estructurar la formación teniendo en cuenta los diversos niveles de competencia de los que parte cada cual.

"Los tipos de usuario van a ser distintos. Uno querrá una ayuda rápida porque está atascado «Quiero poner algo en el aula virtual, cómo lo configuro», o algo mucho más largo" (P2).

"Echo en falta una formación más específica o más de necesidades propias de cada especialidad" (P4).

"El tipo de recursos está ligado al tipo de docencia que tienes entonces, una formación por ámbitos podría resultar interesante. Resulta complejo tener diferentes niveles, algunos lo encuentran muy fácil, otros muy difícil" (G1).

\section{Efecto de la pandemia derivada de la Covid-19 en la percepción sobre la CDD}

Todas las personas entrevistadas manifiestan que la pandemia derivada de la Covid-19 ha tenido una incidencia clara y notable en la docencia universitaria. En relación con la CDD, todos estuvieron de acuerdo en que la pandemia generó una necesidad urgente de incorporar herramientas digitales para continuar con las clases de forma virtual. Al reflexionar respecto a las consecuencias derivadas de esta circunstancia en el nivel de CDD del profesorado universitario, se encuentran dos opiniones diferenciadas. Por un lado, algunos entrevistados sostienen que la autopercepción habrá mejorado, como consecuencia del incremento en el uso de las tecnologías tanto a nivel profesional como también personal.

"De repente se ha hecho en 3 meses lo que se iba a hacer en 3 años. Gente que no tenía ni una webcam, ni había usado una, como la ha tenido que usar para hablar con sus familiares, pues mira nos hemos digitalizado todos" (P2).

"Entre marzo y abril tuvimos que ponernos las pilas. Fue el momento de más catarsis, «tengo que pasar a online, voy a ponerme las pilas» y no sé si eso pudo de alguna manera afectar a la percepción que pudiera tener el profesorado en su momento" (P4).

"Ha sido un proceso muy rápido, de unos meses, donde la gente ha asumido muy rápido las oportunidades que tenía, porque no tenía más remedio. Eso ha 
provocado un aumento de ciertas competencias, relativas al tema de recursos digitales, muy rápida y de mucha gente" (T3).

Sin embargo, algunos entrevistados señalan que entrar en contacto súbito con herramientas y situaciones desconocidas hasta ese momento, ha supuesto una disminución de la autopercepción sobre la propia CDD, y postulan que ese efecto puede haber sucedido igualmente en las personas que cumplimentaron el cuestionario antes de la pandemia.

"Seguro que ha mejorado en muchos aspectos porque muchas veces lo que falta es ponerse a utilizar las herramientas. Es lo que ha pasado en esta inmersión que hemos tenido en la competencia digital, pero al ponerse a hacer cosas, puede que hayan visto carencias en áreas determinadas, en competencias más específicas. Entonces podría variar, se podrían ver más necesidades de formación" (T1).

"En mi evaluación habré puesto todo altito, pero ahora no soy tan positivo. Hay que bajar un poco el nivel, la digitalización se actualiza muy rápidamente y nos quedamos obsoletos en un mes. Esta pandemia de la que hablamos parece que sea de hace 20 años, estamos hablando de meses" (P7).

"Creo que ha supuesto un esfuerzo que no tenía que haber sido tanto si realmente estuviéramos tan bien. Si a estas personas se lo volvieras a preguntar después de esto cómo creen que están, sería curioso. Ahora me he dado cuenta que sé hacer menos cosas, mi visión es que sabía menos de lo que pensaba” (G2).

Respecto a la incidencia en la formación digital del profesorado, según algunos entrevistados, la situación creada por la pandemia puede haber incrementado el interés formativo, ya sea de una manera voluntaria o forzada por la situación.

"La situación ha despertado un poco interés del profesorado de plantearse las cosas de otra manera y de un interés por digitalizar otros aspectos que hasta ahora íbamos a lo más tradicional o más la costumbre, ahora tenemos que estar preparados a situaciones imprevistas y para ya, tienes que tener el conocimiento para hacerlo" (P8).

"Se ha ampliado el perfil, mucha más gente que se ha visto con problemas para organizar sus actividades habituales, para ver cómo organizaban un examen, ver cómo se aclaraban con la docencia, tenían muchas dificultades”(T1).

En algunas de las entrevistas se pone en valor la importancia de la actitud docente y el compromiso profesional como elementos clave para un adecuado aprovechamiento de la formación. 
"Un tema clave es la actitud hacia las nuevas tecnologías. Quien tenga una actitud negativa previa se va a bloquear y por más horas de formación que le demos no vamos a hacer más que bajar su autoeficacia profesional. Es lo que he visto que pasó con esta crisis con los profesores" (G2).

Para concluir, algunos participantes reflexionan sobre la necesidad de incidir en el cambio metodológico o pedagógico del uso de las TD, más allá del conocimiento técnico.

"La parte que veo más complicada es la pedagogía, lo que hemos sufrido ahora es más una pedagogía de emergencia que otra cosa, cada uno se ha acoplado como ha podido. Ahí veo que habría que trabajar más" (T3).

"Si no humanizamos el espacio virtual no funciona, porque es aséptico, muy frío. Normalizar el espacio, es digital pero no es digital, qué tipo de comunidad quiero establecer a través de las pantallas" (P9).

\section{DISCUSIÓN}

En la última década, las universidades han revisado sus planes de digitalización, apostando en algunos casos por la formación digital de la comunidad universitaria. Este es el caso de la UJI, en la cual se desarrollaron dos portales vinculados a la formación docente: (1) la plataforma de autoevaluación de la CDD; y (2) la plataforma de recursos de formación digital. Este artículo se ha centrado en profundizar en la percepción acerca del nivel de CDD, conocer la valoración de la herramienta formativa, así como analizar la posible incidencia de la pandemia de la Covid-19 en la CDD.

En relación con la primera pregunta de investigación, los participantes encuentran que dichos resultados reflejan en gran medida la realidad de la universidad, con un nivel medio de CDD, obteniendo valores más altos en las competencias profesionales y más bajos en las pedagógicas y de empoderamiento de sus estudiantes. Sin embargo, existen algunos puntos de discrepancia, centrados principalmente en la sobreestimación de las puntuaciones. Esto coincide con investigaciones previas, como la planteada por Esteve et al. (2016) quienes apuntan que, si bien la percepción refleja una distribución competencial similar al desempeño real, puede tender a obtener valores algo más altos.

Como línea futura de trabajo, sería interesante triangular los resultados con la percepción del estudiantado sobre la CDD del profesorado, ya que puede brindar información complementaria. En este sentido, los recientes resultados de la Cátedra de Brecha Digital y Buen Uso de las TICs (2020) sobre la CD del estudiantado valenciano, en el cual han participado más de 1700 estudiantes de la UJI, reflejan la demanda del alumnado sobre la necesaria mejora de las capacidades digitales y pedagógicas para la docencia en línea. 
En cuanto a la segunda pregunta de investigación, la plataforma formativa se valoró como muy útil, especialmente al estar vinculada con la herramienta de evaluación diagnóstica previa. En este sentido, las sugerencias de mejora que propusieron los participantes fueron: (1) la posibilidad de disponer de recursos de autoformación asíncronos y abiertos; (2) la especificidad en el buscador para localizar los cursos en función de las distintas áreas de conocimiento, así como la estructura por niveles de complejidad; (3) la opción de disponer de un sistema de valoración o recomendación de los recursos; (4) la visibilidad de la plataforma, lo cual resulta esencial para una adecuada accesibilidad a los recursos formativos; y (5) la posibilidad de interacción con otros usuarios, permitiendo a los docentes intercambiar conocimientos y proponer temáticas formativas de su interés. Siguiendo con los planteamientos de Plomp y Nieveen (2009), estas recomendaciones pueden generalizarse, a modo de principios de diseño, para otras situaciones con un objetivo similar, más allá del presente contexto.

Finalmente, en relación con la tercera pregunta de investigación, si bien los participantes reconocen el esfuerzo realizado tanto por el profesorado como por la propia institución, por mantener la docencia en línea durante este periodo, no hay convencimiento generalizado de que esta situación haya incrementado la percepción sobre la CDD, sino que en algunos casos puede haberse dado justo la posibilidad contraria. Tras la situación vivida, el profesorado ha sido consciente de todas las herramientas y posibilidades didácticas que previamente desconocía, y a consecuencia de ello, su percepción sobre su CDD ha disminuido (Sánchez, 2020). En este sentido, además de reiterar los efectos y limitaciones de las evaluaciones basadas en la autopercepción anteriormente mencionados, sería necesario explorar otros factores de carácter psicosocial, como la actitud hacia las TD (Fernández et al., 2016), o tecnofobias asociadas al género (Castaño, 2008).

En cuanto a las limitaciones de esta investigación cabe destacar el número de informantes participantes en el estudio. Si bien, en la selección se ha buscado la representatividad, como remarca Tessmer (1993), las entrevistas pueden aportar información relevante en la evaluación formativa de una intervención o recurso concreto, pero resulta conveniente contar con un número representativo de participantes que permita la triangulación de la información recogida. $\mathrm{Al}$ respecto se propone para investigaciones futuras dar voz al estudiantado.

Más allá de las limitaciones expuestas, disponer de recursos tanto para la evaluación de la CDD como para la formación digital, supone doble beneficio al profesorado universitario. Por un lado, el proceso de autoevaluación, resulta un elemento de metaformación que propicia la toma de conciencia sobre esta competencia, sobre el metalenguaje que la rodea, y las carencias o necesidades en torno a esta. La plataforma formativa por su parte, permite ir más allá del diagnóstico, facilitando la superación de brechas digitales, centralizando la información y simplificando su búsqueda, proporcionando recursos ajustados a cada necesidad. En cuanto a la Universidad, este tipo de iniciativas ofrecen un gran potencial para 
redirigir sus políticas de formación docente, al obtener información respecto a la CDD de su plantilla, que le permitirá establecer planes específicos de formación inicial y continua del profesorado (Cabero-Almenara et al., 2020) así como tomar decisiones informadas sobre el uso, adquisición o potenciación de recursos y herramientas tecnológicas.

\section{AGRADECIMIENTOS}

La presente investigación se ha realizado con apoyo del Plan de Promoción de la Investigación de la Universitat Jaume I (Ref.: UJI-A2018-09).

\section{REFERENCIAS}

Aalborg University. (2018). Knowledge for the World. https://www.strategi.aau. $\mathrm{dk} /$ digitalAssets/602/602501 040718 digitalstrategy.pdf

Alarcón, R., del Pilar-Jiménez, E., y VicenteYagüe, M. (2020). Development and validation of the DIGIGLO, a tool for assessing the digital competence of educators. British Journal of Educational Technology. http://doi.org/10.1111/ bjet.12919

Athabasca University. (2018). RISE: Athabasca University's Digital Transformation. https://www. athabascau.ca/vice-presidentinformation-technology/ documents/ rise.pdf

Cabero-Almenara, J., Gutiérrez-Castillo, J., Palacios-Rodríguez, A., y BarrosoOsuna, J. (2020). Development of the Teacher Digital Competence Validation of DigCompEdu Check-In Questionnaire in the University Context of Andalusia (Spain). Sustainability, 12(15), 6094. https://doi.org/10.3390/su12156094

Caena, F., y Redecker, C. (2019). Aligning teacher competence frameworks to 21st century challenges: The case for the European Digital Competence Framework for Educators (DigCompEdu). European Journal of Education, 54(3), 1-14. https:// doi.org/10.1111/ejed.12345
Castaño, C. (2008). La segunda brecha digital. Cátedra.

Cátedra de Brecha Digital y Buen Uso de las TICs. (2020). Análisis de las capacidades digitales del estudiantado universitario valenciano. https://bit.ly/37YBWGW

Corbin, J., y Strauss, A. (2015). Basics of Qualitative Research: Techniques and Procedures for Developing Grounded Theory. SAGE Publications, Inc.

Drennan, J. (2003). Cognitive interviewing: Verbal data in the design and pretesting of questionnaires. Journal of Advanced Nursing, 42(1), 57-63. https://doi. org/10.1046/j.1365-2648.2003.02579.x

Durán, M., Gutiérrez, I., y Prendes, M. (2016). Análisis conceptual de modelos de competencia digital del profesorado universitario. RELATEC: Revista Latinoamericana de Tecnología Educativa, 15(1), 97-114.

Esteve, F., Cela, J., y Gisbert, M. (2016). ETeach3D: designing a $3 \mathrm{D}$ virtual environment for evaluating the digital competence of pre-service teachers. Journal of Educational Computing Research, 54(6), 816-839. https://doi. org/10.1177/0735633116637191

Esteve, F., Llopis, M., y Adell, J. (2020a). Digital teaching competence of university teachers: A systematic review of the literature. IEEE Revista Iberoamericana 
de Tecnologías del Aprendizaje, 15(4), 399-406. $\quad$ https://doi.org/10.1109/ RITA.2020.3033225

Esteve, F., Llopis, M., Viñoles, V., y Adell, J. (2020b). El profesorado universitario en la sociedad digital. Diseño de una plataforma de autoevaluación diagnóstica de su competencia digital docente. In XXIII Congreso Internacional EDUTEC 2020. Universidad de Málaga. https://hdl.handle.net/10630/20345

Fernández, J. C., Fernández, M., y Cebreiro, B. (2016). Competencias en TIC del profesorado en Galicia: Variables que inciden en las necesidades formativas. Innovación educativa, 26. https://doi. org/10.15304/ie.26.3256

Fernández-Batanero, J., Montenegro-Rueda, M., Fernández-Cerero, J., y GarcíaMartínez, I. (2020). Digital competences for teacher professional development. Systematic review. European Journal of Teacher Education, 1-19. https://doi.org/ 10.1080/02619768.2020.1827389

Gisbert, M., González, J., y Esteve, F. (2016). Competencia digital y competencia digital docente: una panorámica sobre el estado de la cuestión. Revista Interuniversitaria de Investigación en Tecnología Educativa, o, 74-83. https:// doi.org/10.6018/riite2016/257631

Halász, G. (2019). Designing and implementing teacher policies using competence frameworks as an integrative policy tool. European Journal of Education, ejed.12349. https://doi. org/10.1111/ejed.12349

Hall, R., Atkins, L., y Fraser, J. (2014). Defining a self-evaluation digital literacy framework for secondary educators: The Digilit Leicester project. Research in Learning Technology, 22. http://dx.doi. org/10.3402/rlt.v22.21440

Ion, G., y Cano, E. (2012). La formación del profesorado universitario para la implementación de la evaluación por competencias. Educación XX1, 15(2). https://doi.org/10.5944/educxx1.15.2.141
Krumsvik, R. (2012). Teacher educators' digital competence. Scandinavian Journal of Educational Research, 58(3), 269-280. https://doi.org/10.1080/00313 831.2012.726273

López, C., Benedito, V., y León, M. (2016). El Enfoque de Competencias en la Formación Universitaria y su Impacto en la Evaluación. La Perspectiva de un Grupo de Profesionales Expertos en Pedagogía. Formación Universitaria, 9(4), 1122. $\quad$ https://doi.org/10.4067/So71850062016000400003

Llopis, M., Herrera, M., y Esteve, F. (2020). El profesorado universitario en la sociedad digital. Evaluación de su competencia digital docente. En Congreso Internacional Virtual USATIC, Aprendizaje con TIC. Universidad de Zaragoza. https://zaguan. unizar.es/record/95693

Marín, V., Vázquez, A., Llorente, M., y Cabero, J. (2012). La alfabetización digital del docente universitario en el espacio europeo de educación superior. Edutec. Revista Electrónica de Tecnología Educativa, 39. https://doi.org/10.21556/ edutec.2012.39.377

Marinoni, G., Land H., y Jensen, T. (2020). The Impact of Covid-19 on Higher Education around the world: IAU Global Survey Report. International Association of Universities https://www.iauaiu.net/ IMG/pdf/iau covid19 and he survey report final may 2020.pdf

Marshall, S. (2018). Shaping the University of the Future. Springer Singapore. https:// doi.org/10.1007/978-981-10-7620-6

Mercader, C., y Gairín, J. (2020). University teachers' perception of barriers to the use of digital technologies: the importance of academic discipline. International Journal of Educational Technology in Higher Education, 17(4), 1-14. https:// doi.org/10.1186/s41239-020-0182-x

Orozco, G., Cabezas, M., Martínez, F., Delgado, J., y Solís, M. (2016). Determining factors in acceptance of ICT by the University faculty in their teaching 
practice. ACM International Conference Proceeding Series, 02-04-November-2016 (pp. 139-146). $\quad$ https://doi. org/10.1145/3012430.3012509

Pettersson, F. (2018). On the issues of digital competence in educational contexts a review of literature. Education and Information Technologies, 23(3), 10051021. https://doi.org/10.1007/s10639017-9649-3

Plomp, T., y Nieveen, N. (2009). $A n$ introduction to educational design research. Netherlands Institute for curriculum development (SLO).

Pozos, K., y Tejada, J. (2018). Competencias Digitales en Docentes de Educación Superior: Niveles de Dominio y Necesidades Formativas. Revista Digital de Investigación en Docencia Universitaria, 12(2), 59-87. https://doi. org/10.19083/ridu.2018.712

Rangel, A., y Peñalosa, E. A. (2013). Alfabetización digital en docentes de Educación Superior. Construcción y prueba empírica de un instrumento de evaluación. Pixel-Bit-Revista de Medios y Educación, 43, 9-23. https://doi. org/10.12795/pixelbit.2013.i43.01

Redecker, C., y Punie, Y. (2017). European Framework for the Digital Competence of Educators. DigCompEdu. European Commission, Joint Research Centre (JRC).

Rodríguez-Gómez, G., Gil-Flores, J., y García-Jiménez, E. (1999). Metodología de la investigación cualitativa. Ediciones Aljibe.

Ruè, D. (2015). El desarrollo profesional docente en Educación Superior: agenda, referentes y propuestas para su adopción. REDU. Revista de Docencia Universitaria, 2015, 13, 217-236. https:// doi.org/10.4995/redu.2015.5461

Saldaña, J. (2015). The Coding Manual for Qualitative Researchers. Third Edition. SAGE Publications Ltd.
Sánchez, J. (2020). Intervención en línea para el aumento de la autoeficacia en habilidades docentes por internet ante la contingencia del covid-19. Enseñanza \& Teaching, 38, 1-2020, 125-145. https:// doi.org/10.14201/et2020381125145

Teddlie, C., y Yu, F. (2007). Mixed methods sampling: A typology with examples. Journal of Mixed Methods Research, 1(1), 77-100. https://doi. org $/ 10.1177 / 1558689806292430$

Tessmer, M. (1993). Planning and conducting formative evaluations: Improving the quality of education and training. Kogan Page.

Tourón, J., Martín, D., Navarro Asensio, E., Pradas, S., e Íñigo, V. (2018). Validación de constructo de un instrumento para medir la competencia digital docente de los profesores (CDD). Revista Española de Pedagogía, 76(269), 25-54. https:// doi.org/10.22550/REP76-1-2018-02

Universitat Jaume I. (2020). Plan UJI Digital. https://ujiapps.uji.es/ade/rest/storage/RQW7SHVBKRFYLYMSRJOCJIMM7KZBQR3A

Université Catholique de Louvain. (2015). Digital strategy at UCLouvain: openness matters. https://dial.uclouvain.be/pr/ boreal/object/boreal:195896

Université de Genève. (2019). Digital Strategy Action Plan. https:// w w w.unige.ch / n u m erique/ files/2115/4754/2835/15012019 Plan daction.pdf

University of Auckland. (2018). Digital Strategy 2018-2020. https://cdn. auckland.ac.nz/assets/auckland/aboutus/the-university/official-publications/ other-publications/digital-strategyo1feb-2018-slt-endorsed-final.pdf

University of Bergen. (2016). Digitalisation that shapes society. http://ekstern.filer.uib.no/ledelse/ digitaliseringsstrategi 2016-22 ENG.pdf 


\section{PERFIL ACADÉMICO Y PROFESIONAL DE LOS AUTORES}

Virginia Viñoles-Cosentino es doctoranda en Educación por la Universitat Jaume I, desarrolla su investigación en el grupo GREAT (Enseñanza, Aprendizaje y Tecnología) y Grupo EDUBOT (Innovación en Robótica Educativa y Pensamiento Computacional). Licenciada en Psicología, Postgrado en Digital Learning y Máster en Psicología de las Organizaciones. Experiencia en gestión de cambio en proyectos de transformación digital en el sector público y privado. Sus intereses de investigación se centran en: competencias digitales, formación docente, tecnología educativa. https://orcid.org/0000-0002-9048-5827

E-mail: vvinoles@uji.es

Francesc M. Esteve-Mon es comisionado de la rectora para la coordinación del gabinete y el impulse digital, y profesor Ayudante Doctor en el Departamento de Pedagogía de la Universitat Jaume I, España. Centra su docencia en el grado de Educación Infantil. Diplomado en Magisterio, Licenciado en Psicopedagogía, y Máster y Doctor en Tecnología Educativa. Miembro del grupo de investigación en Enseñanza, Aprendizaje y Tecnología (GREAT), desarrolla su investigación en competencia digital, formación docente, e investigación-basada en el diseño. https:// orcid.org/0000-0003-4884-1485

E-mail: festeve@uji.es

María Ángeles Llopis-Nebot es profesora Asociada en el Departamento de Pedagogía de la Universitat Jaume I, España. Centra su docencia en el grado de Educación Infantil y coordina las estancias de prácticas. Doctora en Educación por la Universitat Jaume I, desarrolla su investigación en el grupo GREAT (Enseñanza, Aprendizaje y Tecnología) y EDUBOT (Grupo de Innovación en Robótica Educativa y Pensamiento Computacional). Sus intereses de investigación se centran en el pensamiento reflexivo y computacional, y en la competencia digital. https://orcid. org/0000-0002-9192-7076

E-mail: mllopis@uji.es

Jordi Adell-Segura es director del Centro de Educación y Nuevas Tecnologías (CENT) y profesor Titular en el Departamento de Pedagogía de la Universitat Jaume I, España. Doctor en Filosofía y Ciencias de la Educación, es el coordinador del grupo de investigación en Enseñanza, Aprendizaje y Tecnología (GREAT). Centra su docencia e investigación en tecnología educativa. Ha participado en diferentes paneles y comités asesores en Tecnología y Educación. https://orcid.org/oooo0003-4369-6485

E-mail: jordi@uji.es 


\section{DIRECCIÓN DE LOS AUTORES}

Universitat Jaume I

Av Sos Baynat, s/n 12071

Castelló de la Plana

España

Fecha de recepción del artículo: 14/12/2020

Fecha de aceptación del artículo: 02/02/2021

Fecha de aprobación para maquetación: 10/02/2021 